\title{
La construction du sujet : entre complémentation et sélection
}

\author{
Jacques Evouna \\ Université du Cameroun \\ jacquesevouna@yahoo.fr
}

\begin{abstract}
Résumé. Cette contribution s'intéresse à la relation qui lie le sujet au verbe. La complexité des questions abordées ici les inscrit au cœur des problématiques actuelles de la linguistique française. La forme du verbe (en termes de variations) permet-elle de montrer que le sujet en est une propriété catégorielle ? On suppose que la construction de cette position fonctionnelle s'explique moins par ce qu'en dit la grammaire (traditionnelle) - la transmission des marques formelles (donc l'accord) - que par la sélection de ses traits catégoriels. La démarche s'appuie sur le module chomskyen X-barre qui permet de postuler le déplacement du sujet de Spéc, V" vers Spéc, I". Quelle est cependant la justification de ce déplacement ? L'objectif visé est de proposer une alternative d'analyse à l'uniformité linéaire et unidirectionnelle du modèle générativiste dont la tendance est à l'unification des syntagmes constitutifs de la phrase. (2) À partir de l'érection en têtes de projections des catégories nominales et des catégories verbales, l'immanentisme catégoriel apparaît comme le principe explicatif de la sélection catégorielle du nombre par l'inflexion. (3) On envisage alors de poster le sujet autre part que sous Spéc, I", c'est-à-dire $\mathrm{d}$ 'en faire une projection maximale à part entière.
\end{abstract}

\begin{abstract}
The present paper investigates into the relation between the subject and the verb. The complexity of questions herein raised places them at the core position of current research issues in French linguistics. Is the form of the verb (from the point of view of variations) proof enough that the subject is a categorial property of it? It is assumed that the construction of this functional position is less accounted for by what (traditional) grammar, the transmission of formal markers (among which, agreement) than by the selection of their categorial specificities. The approach is based on Choamskyan X-bar theory which enables the movement of the subject Spec, V' towards the Spec, I'. What however accounts for this movement? The intention is to propose an alternative analysis for the linear and unidirectional uniformity of the generativist model whose tendency is the unification of phrases making up the sentences. (2) Basing on the setting up of nominal and verbal categories as head words, categorial immanentism stands out as the principle underlying categorial selection of the number by the inflexion. (3) It is hence deemed wise to posit the subject under a different appellation than Spec, I', that is to make it a full maximal projection.
\end{abstract}




\section{Introduction}

Suivant l'une des règles générales de la tradition grammaticale, le sujet commande l'accord du verbe. En d'autres termes, le sujet transmet au verbe ses marques formelles, de la même manière qu'à l'adjectif qualificatif. Cependant, les exemples (1) suivants :

Les enfants malades mangent.

Les filles intelligentes étudient leurs leçons.

montrent que les marques formelles de l'adjectif, qu'il tient manifestement du nom, sont différentes de celles que porte le verbe. Quoique l'on conçoive le sujet comme «le mot qui donne sa loi au verbe », (Vaugelas) ou que l'on admette l'idée de la transmission des marques du sujet au verbe, (Riegel et al, 1993) ces configurations (2) sont illicites :

*Les enfants maladent manges.

*Les filles intelligentent étudies leurs leçons.

Les grammairiens sont en revanche unanimes sur la régularité des ces autres (3) :

La cérémonie des fiançailles s'est déroulée merveilleusement.

Les collègues que j'ai invités tardent à arriver.

Les disparités tiennent probablement au fait que l'application des catégories du genre et du nombre se limite au substantif. (Baylon et Fabre, 1978: 71) Au-delà de ces différences formelles, la logique de l'accord compte des justifications théoriques. La grammaire générative considère par exemple que le sujet occupe le domaine de spécification de l'inflexion; ce qui en fait une propriété catégorielle du verbe. Le sujet vérifie-t-il les traits du verbe, de la même manière que l'article vérifie les traits du nom ? Que les propriétés morphologiques et fonctionnelles du spécificateur restent à décrire n'empêche pas de noter l'unité catégorielle du nom et de son article. Le couple sujet-verbe s'illustre cependant par une disparité catégorielle. Cette différence rend nécessaire l'étude des modalités d'association des catégories nominales et des catégories verbales.

\section{La flexion verbale : une marque d'accord?}

Cette section de l'article examine les conditions et les implications de la flexion verbale sur l'analyse des règles d'accord du verbe avec le sujet. Selon les observations générales de Grevisse et Goosse (2004 : 1315), l'accord concerne le verbe conjugué. Malgré cette restriction, quelques grammairiens confèrent un pouvoir plus grand au sujet. Maingueneau (1999 : 31), par exemple, parle de la prééminence du sujet qui se traduit par le phénomène d'accord. L'on peut pourtant parler de sujet pour l'infinitif. Cette éventualité autorise-t-elle à lier les variations du verbe à la forme d'un sujet ? Y a-t-il accord du verbe fini ? Ainsi commencent à surgir des restrictions à l'affirmation que le sujet transmet au verbe ses marques de personne, de nombre et parfois de genre. En effet, « Cela ne peut s'appliquer ni à l'infinitif ni au participe présent, puisqu'ils sont invariables. » (Grevisse et Goosse 2004 : 305) Cet état des choses ne laisse guère envisager qu'une piste à explorer : puisque le sujet s'avère étranger à la forme du verbe, il faut apporter la preuve que les marques formelles de celui-là influencent la flexion de celui-ci. Cet impératif vaut, à la fois, pour les formes unies et pour les formes éclatées $1 \mathrm{du}$ verbe. 


\subsection{La structure catégorielle des formes verbales simples}

Quoique brièvement, ce point examine les lois régissant l'élaboration et l'accord des formes verbales simples. La démarche, qui consiste à discerner les catégories grammaticales impliquées dans la conjugaison du verbe, partira du rappel de la règle générale : «le verbe s'accorde en nombre et en personne avec le sujet. » (Riegel et al. 1993 : 345) Une question, pourtant, peut suffire à éprouver la validité prédictive de cette règle : l'inflexion compte-t-elle au nombre des propriétés catégorielles du nom, ou même d'un infinitif en position de sujet ? À faire une telle affirmation, on expose l'analyse à une nouvelle difficulté : expliquer que l'adjectif ne s'accorde pas en personne. Sachant que les catégories nominales sont constituées du genre et du nombre, est-on fondé à inscrire le nombre sur la liste des catégories verbales? Que l'on considère simplement la morphologie d'un verbe conjugué, quel(s) élément(s) renvoie(nt) précisément au nombre ? L'un des arguments avancés pour justifier un défaut d'adéquation descriptive fait état d'une situation d'amalgame dans les désinences verbales. La conclusion, évidente de ces analyses, consacre alors la difficulté, voire l'impossibilité de démêler les différentes catégories grammaticales engagées.

Le fait que l'implication de certaines catégories dans le phénomène d'accord en exclue certaines autres pose la question-même de son cadre d'application. La grammaire admet l'idée d'un accord du verbe en nombre, en personne et même en genre avec le sujet. En termes d'accord, l'adjectif porte-il des marques catégorielles qu'il ne tienne du nom ? Faut-il nécessairement interpréter les flexions verbales, c'est-à-dire (les variations dues à) la conjugaison comme une mise en œuvre de l'accord ? Cela suppose, hypothèse défendue par la tradition grammaticale, la transmission des marques formelles du sujet au verbe. La solution consiste peut-être à cerner puis à montrer, dans le cas de chaque catégorie verbale, son rapport avec le sujet. L'accord porte généralement sur les éléments terminaux du mot. Pour ce qui concerne spécifiquement le verbe, les désinences représenteraient les marques de son accord. Cependant, un même verbe conjugué à des temps différents ne «s'accorde » jamais de la même façon avec le même sujet (4) :

Je songeai ; je songe ; je songeais.

Nous songeâmes ; nous songeons ; nous songions.

La thèse de l'accord verbal, pour convaincre, doit fournir une explication à ces disparités. Il semble en revanche exister une corrélation entre les variations des désinences et celles du temps. En outre, la perception que l'on a de l'accord dans cette réflexion est qu'il s'agit d'un phénomène de récursivité des marques formelles. Cette conception s'applique-t-elle aux catégories verbales ? Leur configuration semble plutôt suivre un principe de discontinuité. Ainsi, les désinences font partie intégrante d'un système corrélatif. Cela implique de les considérer autrement que comme des marques d'accord. C'est en effet une démarche coûteuse que de devoir démontrer que « $\mathrm{s}$ » et même souvent « $\mathrm{X}$ » $\mathrm{s}$ 'accordent avec « je », ou « tu ». Elle le paraît moins de les décrire comme des éléments constitutifs d'une corrélation. 
L'analyse grammaticale se trouve régulièrement confrontée à un problème d'identification des faits. Par exemple, Grevisse et Goosse (2004 : 1316) estiment que la forme « il » est un pronom qui « commande l'accord du verbe, lequel reste donc au singulier ». Il y a, en outre, quelques exceptions troublantes : «le verbe ayant pour sujet le pronom relatif «qui » se met au même nombre et à la même personne que l'antécédent de ce pronom. » (1317) Il faut néanmoins relever, pour rapprocher «qui » des «pronoms personnels sujets », sa remarquable insensibilité à la différence de nombre, mais aussi sa contiguïté avec la finitude temporelle du verbe. L'option la moins risquée consiste à classer ces éléments comme des marques de la conjugaison. Dans le prolongement des analyses de Kayne, on supposera que «qui » résulte d'une opération de fusion (Laenzlinger 2006), celle de la conjonction (que) et de la rémanence catégorielle (indice probablement consécutif à un effacement) de la personne grammaticale (i). Pour la plupart, les éléments de la série dite « des pronoms personnels sujets » sont en réalité des occurrences de la personne grammaticale. Le sujet est-il aussi essentiel au verbe qu'on l'affirme ? Que l'on place, pour y voir clair, « le pronom il » au centre d'une brève analyse (5) :

Il me vient une idée.

$I l$ arrive des gens.

Il me faut des hommes de main.

Il pleut.

Quelques points autorisent à considérer, contre ce qui est habituellement admis, que l'emploi des verbes «arriver», «falloir» et «pleuvoir» se fait sans sujet dans les énoncés ci-dessus. Le questionnement auquel la pratique scolaire recommande de recourir pour identifier le sujet disqualifie la forme «il» de ce rôle (6) :

* Qu'est-ce qui me vient une idée?

* Qui arrive des gens?

* Qu'est-ce qui me faut des hommes de main?

* Qui pleut?

De plus, la démarche qui oppose un «sujet apparent » à un «sujet réel » débouche sur une alternative. Il y a, d'une part, la possibilité (6b) souvent évoquée et exploitée de ramener en début d'énoncé l'item pressenti dans le rôle du sujet. Elle ressortit cependant à une conception éminemment sémantique du sujet, au sens de «l'être ou la chose qui fait ou qui subit l'action ou qui est dans l'état exprimé par le verbe. » (Riegel et al. 1993) Mais, d'autre part, les choses ne sont pas toujours aussi simples qu'elles peuvent souvent le paraître, puisque la manipulation est impossible, les résultats illicites (6c, d) ou douteux (6a) :

? Une idée me vient.

Des gens arrivent.

* Des hommes de main me faut.

* pleut.

Pour la suite de la réflexion, il faut même se demander si la possibilité d'interpréter les positions en cause comme des positions d'objets est à écarter complètement. Les questions suivantes semblent conformes aux méthodes de pronominalisation mises en œuvre dans les grammaires pédagogiques (7) :

Que me vient-il / Il m'en vient une.

Qui arrive-t-il ? / Il en arrive.

Que me faut-il ? / Il m'en faut. 
Pour récapituler, deux situations se présentent de part et d'autre du verbe : à gauche, l'incapacité de la personne absolue, d'univers ou sans référence (l'impersonnel), à remplir un rôle syntagmatique auprès du verbe ; à droite, vraisemblablement l'occurrence d'un complément. Faut-il maintenir qu'il y a accord du verbe ici, c'est-à-dire avec un sujet qui n'en paraît pas un ? La question s'étend aux rapports qui lient les particules préverbales (Muller, 2002) que la grammaire appelle pronoms personnels sujets et le verbe. Les processus de relativisation ou de clivage mettent en lumière la fragilité des restrictions fonctionnelles qui caractérisent les pronoms dits compléments. Leur capacité à remplir les positions fonctionnelles, à la fois, de sujet ou de complément est démontrable.

L'état des choses ainsi décrit s'apparente à une situation de relative indécision. Malgré ce qu'on en dit (Riegel et al. 1993), la fusion n'empêche pas de manière absolue d'identifier séparément les marques formelles constitutives de la morphologie du verbe fini. Par exemple, [ra] est attesté comme la marque distinctive du futur, [ai] comme celle de l'imparfait. Le mode, la finitude temporelle et le type de verbe sont, entre autres éléments de la flexion verbale, ceux qu'il serait nécessaire à une démarche descriptive de cet ordre de prendre en compte. Pour les deux temps verbaux ainsi pris en exemple, la question à résoudre concerne la nature précise des morphèmes terminaux : «partira-i », «partira-s », «partira-ø »; « $\operatorname{march}(\mathrm{e}-\mathrm{a}) \mathrm{i}-\mathbf{o n s}$ », «march(e-a)i-ez », « march(e)-ai-ent ». Le problème est probablement né, d'une part, des difficultés de la grammaire à établir un élément comme marque formelle de la catégorie du nombre dans la morphologie du verbe. D'autre part, la grammaire (Bonnard 1988) tient le nombre et la personne comme des catégories indissociables (et même le temps) - le sont-ils ? On dit bien «le genre nominal », «le nombre nominal », ou «le temps verbal», «le mode verbal». À quel titre dit-on cependant différemment «première personne du singulier» ou «troisième personne du pluriel »? Combien va-t-on distinguer de singuliers dans les formes «je mange, tu manges, il mange », ou de pluriels dans «nous voyons, vous voyez, ils voient»? On supposera que seul un item lexical peut servir de support à une catégorie grammaticale. En d'autres termes, la personne, catégorie grammaticale, ne peut servir de support au nombre, une autre catégorie grammaticale. L'opposition de nombre (singulier pluriel) manque donc de pertinence pour le verbe. Il faut réserver respectivement au nom le nombre et au verbe la personne grammaticale. Simplement donc, le verbe se conjugue en personne. Ainsi la flexion verbale se distingue-t-elle de l'accord. Y a-t-il une explication plus probante à fournir à ce sujet ?

\subsection{Les formes éclatées : un procès disjoint des catégories verbales}

Le débat sur les questions relatives à l'accord du participe passé se heurte à une première difficulté liée à l'identité de la forme en cause, qui reste relativement floue. Le participe passé est-il un verbe, un adjectif ou l'un et l'autre à la fois ? Grevisse et Goosse indiquent, en faveur du statut verbal du gérondif et du participe, qu'ils sont susceptibles «1) d'avoir des compléments d'objets et d'autres compléments spécifiques au verbe ; - 2) d'être mis au passif ; - 3) d'exprimer certaines nuances temporelles. » (2004 : 1306) L'autre point de cet exposé des faits présuppose que la forme basique du participe est active. Autrement dit, le statut du passif est adjectival ; ce qui le rend sensible aux catégories nominales.

La grammaire traditionnelle fait cependant état d'une sorte de mitoyenneté morphologique entre le statut verbal et le statut adjectival, en attribuant le nom de participe passé à des formes qui correspondent, à plusieurs égards, à une toute autre description. Pour cette question comme pour d'autres, cette grammaire fait preuve d'une certaine indécision. Elle admet pour certains verbes la possibilité d'avoir deux «participes passés » : «su, ayant su », « allé, étant allé », etc. Certains autres, les verbes pronominaux en l'occurrence, ne peuvent guère avoir qu'un participe passé de forme éclatée : «s'étant promené », «s'étant entendu », «s'étant uni ». Où est la règle et où se trouve l'exception ? À la marge de cette indécision, Baylon et Fabre (1991 : 149) prennent le parti de parler de «participe de forme composée » plutôt que de participe passé. Dans le prolongement de ces deux linguistes, on a identifié le suffixe -ant comme la forme fondamentale du participe, présent et passé. L'absence de ce suffixe est une raison pour envisager une autre nature. Parler de «participe passé » est plutôt inapproprié en cas de conjugaison, puisqu'on affaire à une forme verbale éclatée, constituée d'un auxiliaire fini et du procès verbal. 
L'on s'abstiendra à ce propos de dire, ainsi que sont accoutumés à le faire les grammairiens, "forme composée ». Car cette expression suppose que l'on soit en mesure d'établir une différence entre les éléments constitutifs, c'est-à-dire le verbe et son auxiliaire. Le "participe passé » et l'auxiliaire doivent être traités également, puisqu'ils sont unis par les mêmes propriétés. Il est coûteux de les envisager séparément, c'est-à-dire d'évoquer le participe passé ici et l'auxiliaire là. Inutile, en outre, de s'attarder sur l'accord de l'auxiliaire dont les problèmes correspondent, point pour point, à ceux évoqués pour les formes verbales synthétiques. Reste donc à aborder la question du procès verbal accompli.

À la suite d'Audet (1996), un bref rappel suivi d'un rapide examen des règles générales d'accord de ce qui apparaît dans les grammaires morphologiques sous l'étiquette de participe passé permettront d'établir certaines distinctions. Ces règles, au nombre de trois, sont empruntées à Grevisse et Goosse :

Le participe passé employé sans auxiliaire ou avec l'auxiliaire être s'accorde comme un adjectif qualificatif. (2004:1331)

Le participe conjugué avec avoir s'accorde en genre et en nombre avec son objet direct quand cet objet le précède ; - il ne varie pas (ce qui est le cas le plus fréquent) s'il est suivi de son objet direct ou s'il n'a pas d'objet direct. (2004 : 1332)

a) Quoique les verbes pronominaux se conjuguent avec l'auxiliaire être, ils peuvent avoir un complément d'objet direct, et la tradition grammaticale veut que le participe s'accorde avec ce complément si celui-ci le précède. (2004 : 1343)

b) Quand il n'y a pas d'objet direct (2007 : 1344)

$1^{\circ} \mathrm{Si}$ le pronom réfléchi est objet indirect, le participe reste invariable

$2^{\circ} \mathrm{Si}$ le pronom conjoint est inanalysable, le participe passé s'accorde avec le sujet.

La première de ces trois règles ignore les différences, du reste considérables, entre d'une part l'auxiliaire « être » et la copule «être » et, d'autre part, le procès accompli et l'attribut ou même l'actif (8a) et le $\operatorname{passif}^{2}(8 b)$ :

L'enfant est parti.

L'enfant est puni.

Cela conduit à deux remarques. L'auxiliaire «être » ne peut être précédé d'un autre auxiliaire (9a), à la différence de la copule $(9 b)$ :

* L'enfant a été parti.

L'enfant $a$ été puni.

Avec une économie considérable de tournures embarrassantes, la reconnaissance du statut de verbe au procès accompli aide à cerner l'esprit de la deuxième règle : le verbe de forme éclatée, cessant d'un coup de s'accorder avec le sujet, reçoit désormais les marques formelles du complément d'objet direct qui le précède.

La troisième règle n'est pas moins problématique. Les questions qu'elle suscite se rapportent précisément à l'emploi exclusivement actif des verbes pronominaux. Qu'est-ce qui, en outre, fonde la différence d'accord entre le verbe de forme concentrée et le verbe de forme éclatée ? L'accord implique-t-il une ou plusieurs catégories? Le traitement de ces questions, et celui de bien d'autres encore, reste fortement influencé par l'émiettement conceptuel du phénomène d'accord. L'analyse syntaxique doit encore justifier l'accord en genre et en nombre de l'actif auxilié ou verbe éclaté, c'est-à-dire distinguer l'actif du passif. Inopérant pour le passif, le principe d'éclatement verbal est rentable pour établir cette différence.

La suite de l'article va s'atteler à examiner la forme du sujet sous l'angle de la grammaire générative, à partir notamment de l'application raisonnée du module chomskyen X-barre. Il y sera principalement question de décrire séparément les domaines syntagmatiques du sujet et du verbe avant d'envisager l'étude des relations qui se tissent entre eux. 


\section{La dérivation syntagmatique : hiérarchie de projections maximales}

La grammaire générative reconnaît au verbe la capacité d'occuper la position tête d'une projection maximale, tandis qu'elle la dénie au (pro-nom) sujet. Ce dernier ne constitue-t-il pas un SN ? N'est-il pas précédé du spécificateur (l'article) à propos duquel on affirme qu'il vérifie les traits du sujet (nom) ? Le statut de ce dernier apparaît donc flou. Cette grammaire fait ensuite une différence entre les objets et les sujets. Les premiers forment des SN; les seconds en sont incapables. En fait, explique-t-on dans cette théorie, le spécificateur est le plus souvent un élément de rang zéro, pas une projection maximale. Comment considérer autrement que comme une violation de ce principe l'érection relativement récente en un DP (Determiner Phrase) du sujet, initialement Spéc, I' et en provenance de Spéc, V' ? À partir de la discussion de la structure syntagmes, cette partie veut montrer, pour la langue française, que le syntagme est une hiérarchie de projections maximales, c'est-à-dire des paradigmes, que la phrase est une hiérarchie de syntagmes. Le premier niveau de description des items fonctionnels dont le module X-barre permet de rendre compte est donc paradigmatique. L'un des tout premiers problèmes à aborder se rapporte à l'analyse traditionnelle proposée pour SN et SV :

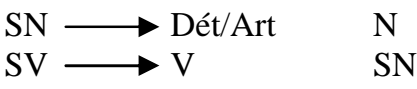

La relation entre Dét et $\mathrm{N}$ est-elle identique à celle qui s'établit entre V et SN ? Il est évident que non. Le nom est précédé de son actualisateur. Qu'en est-il du verbe ? Plus conséquente sur ce point, la grammaire générative formalise un système en deux étapes :

$\begin{array}{lll}\mathrm{N}^{\prime \prime} \longrightarrow \text { Spéc, } \mathrm{N}^{\prime} ; \mathrm{N}^{\prime} \longrightarrow & \mathrm{N} & \text { Compl. } \\ \mathrm{V}^{\prime \prime} \longrightarrow \text { Spéc, } \mathrm{V}^{\prime} ; \mathrm{V}^{\prime} \longrightarrow & \mathrm{V} & \text { Compl. }\end{array}$

Ce qui retient l'attention ici, c'est le spécificateur. Pour le nom, la grammaire générative liste articles et adjectifs déterminatifs. Quant au verbe, l'analyse fait du sujet le spécificateur du verbe. La définition du spécificateur comme propriété catégorielle de la position tête justifie-t-elle l'association de ces éléments ? L'identité du sujet doit être précisée. L'indécision autour de son statut, consécutive au modèle traditionnel qui ignore la gauche du verbe, est telle, en effet, que l'analyse met davantage l'accent sur les déterminants du nom et sur les compléments du verbe. La description de la gauche nominale et de la gauche verbale va s'avérer cruciale pour l'analyse de la construction du sujet.

\subsection{Le syntagme nominal : la fusion du genre et du nombre}

La constitution des syntagmes en français est soumise à des conditions catégorielles précises que d'autres langues, à l'exemple de l'anglais, semblent ignorer et qu'il incombe à l'analyse d'élucider. Aussi envisage-t-on, pour la langue française, de formaliser deux syntagmes. Progressivement, l'on verra se manifester, à l'intérieur d'une même structure, l'incompatibilité des catégories nominales et des catégories verbales. Le syntagme sera décrit comme la superposition de trois projections maximales. Il y a deux projections catégorielles dont l'une est première et l'autre seconde. Il y a ensuite une projection lexicale. On tend à s'écarter de l'attitude générativiste qui consiste à parler systématiquement de phrase, pour faire du principe d'endocentricité l'exclusivité des classes syntaxiques autonomes. On verra, de ce point de vue, que les projections lexicales servent d'hôtes aux projections catégorielles.

L'intérêt des linguistes pour une catégorie comme le genre ne doit pas avoir la même pertinence dans toutes les langues (Vendryes, $1929: 107)^{3}$. Par exemple, son incidence est plus marquée en français qu'en anglais. Les différences restent cependant infimes dans le traitement linguistique réservé à cette catégorie. On propose dans ce qui suit de décrire la projection catégorielle du genre.

Le spécificateur est une propriété catégorielle de la position tête. Les analyses d'inspiration générative admettent régulièrement que les déterminants occupent le domaine de spécification du nom (considération étendue à l'article). Cette approche soulève une première question: le spécificateur représente-t-il deux plutôt qu'une propriété catégorielle de la position tête ? Peut-on, s'appuyant sur cette analyse, envisager le principe d'un syncrétisme catégoriel dans le domaine de spécification d'une tête qui 
compte plusieurs propriétés catégorielles ? L'article, en guise de réponse, sépare le genre du nombre du nom. Il faut par conséquent supposer l'existence de deux spécificateurs. On a manifestement affaire à un cas de cumul, au sens que Laenzlinger (2006) donne au mot « fusion ». Il reste néanmoins à en identifier et à en distinguer les positions têtes. Association du clitique du genre et de la cataphore du nombre, l'article est le produit d'une fusion de deux spécificateurs (Spéc, Gen" et Spéc, Num"). Comme le genre, le nombre encadre le nom. Mais par son hétérogénéité discontinue, le genre se distingue du nombre qui se caractérise par une homogénéité récursive.

La vérification du nombre du nom incombe par ailleurs à la position Spéc, Num". C'est la cataphore du nombre qui joue le rôle de spécificateur dans ce cas :

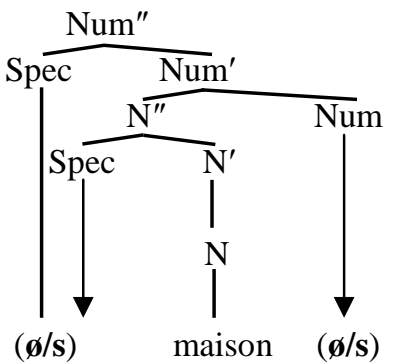

Figure 1 : La récursivité du nombre

Quant à la projection du genre, il lui sera affecté le symbole Gen". Le genre est une catégorie extrinsèque au nom (contra l'idée de l'inhérence du genre émise par Riegel et al. 1993 : 150 et partagée par Grevisse et Goosse, 2004 : 705). Son fondement est physiologique, précisément «la distinction des sexes. » (Baylon et Fabre, 1978 : 65) De forme corrélative, le genre se superpose au nombre mais encadre le nom :

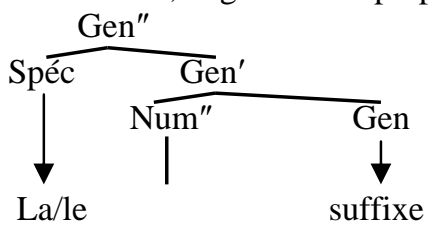

Figure 2 : La discontinuité du genre

La projection du genre inspire une remarque : le genre n'est pas un mot en français, mais une catégorie discontinue. Cela vaut aussi pour le spécificateur lui (imparfaitement l'article), qui fusionne en réalité un clitique $^{4}$ et la cataphore du nombre. Il joue un rôle de vérification. Ainsi, «la» et «le»vérifient respectivement le féminin et le masculin du nom. Si l'être s'identifie de visu comme mâle ou femelle, le nom n'est a priori ni masculin, ni féminin. Seulement, l'information relative au genre n'est généralement pas disponible du fait du suffixe de genre qui n'apparaît pas systématiquement dans la formation de tous les noms de la langue. Une part de l'arbitraire du signe linguistique trouve donc probablement une explication dans l'immanentisme du genre.

La superposition de ces projections aboutit non pas à une phrase, mais à un syntagme. La représentation ci-dessous ne manquera pas, ainsi que le feront sans doute celles qui vont suivre, d'attirer l'attention. Les nœuds s'y coupent, en totale rupture avec l'orthodoxie générativiste qui réserve les cas d'exception aux constituants en incise. Les nœuds préterminaux, souvent, ne comporteront pas de feuille. S'ils sont maintenus, c'est parce qu'ils rendent compte de la structure réelle des items et de leurs relations, c'est parce que, surtout, leur économie constitue un risque pour la cohésion de l'analyse.

On s'éloigne considérablement des analyses (Miller 1992) qui laissent le choix de rattacher l'article au niveau le plus élevé ou au niveau terminal (avec le nom) du syntagme. À se fier à l'observation immédiate, on pourrait voir le nom comme un ajout (élément incident qui induit l'inversion du complément d'une projection maximale). Se trouve-t-il un argument qui permette de démontrer qu'une projection maximale lexicale peut s'interpréter comme complément ou ajout d'une tête catégorielle ? Une telle analyse est erronée, parce que ni le nombre ni le genre ne sont des mots pleins, mais simplement des marques formelles. La bonne solution consiste à supposer que les ajouts s'effectuent dans deux 
directions: de haut en bas pour les ajouts de type lexical, et de bas en haut pour les ajouts de type catégoriel. En d'autres termes, c'est d'abord le nombre qui se superpose au nom, puis le genre. Ce dernier, en outre, est incident entre le nom et le nombre; ce qui explique finalement que les nœuds se coupent.

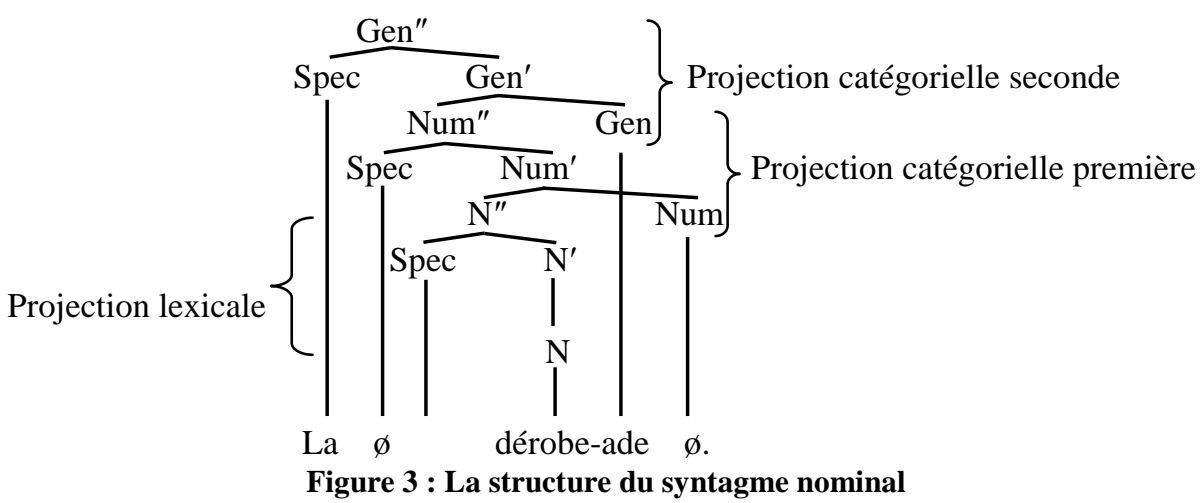

Ainsi, le nom est un item de forme «bicatégorielle ». La vacuité de son domaine de spécification sur cette figure contredit l'idée que «l'article » spécifie le nom.

L'accord est un cadre où la récursivité catégorielle se généralise au genre. Il y a un enjeu à séparer la rection catégorielle établie par des marques formelles - la manifestation du phénomène d'accord tient à la récursivité des marques formelles du genre (AgrGen") et du nombre (AgrNum") - de la dépendance syntaxique (subordination) qui impliquent les items catégoriels et fonctionnels. Le fondement de cette distinction se trouve dans la typologie des projections. Elles sont de type catégoriel (pointillé) ou lexical (trait continu). La projection d'un syntagme dépendant (traits interrompus) y trouve également explication. Ces raisons justifient l'accord de l'adjectif avec le nom support. L'accord signifie donc la récursivité des indices catégoriels. La cohésion catégorielle et l'unité syntaxique ${ }^{5}$ des projections lexicales $\mathrm{N}^{\prime \prime}$ et Adj" dépendent pour l'essentiel du rôle structural des marques catégorielles du genre et du nombre :

Gen"

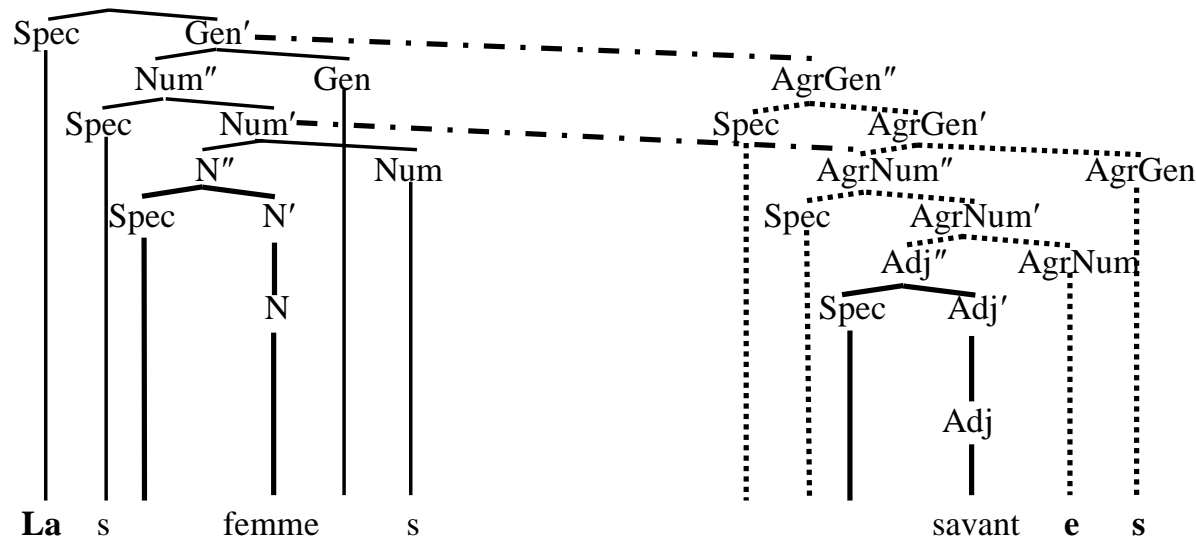

Figure 4 : L'accord de l'adjectif ou la récursivité des catégories nominales

Ainsi, la dérivation syntagmatique des paradigmes du nom et de l'adjectif dépend étroitement des projections catégorielles, c'est-à-dire le genre et le nombre. Mais exclu le féminin, le genre n'a pas toujours de marque spécifique (Baylon et Fabre, 1991). La position tête de la projection du genre est souvent vide. Le genre n'en reste pas moins une catégorie de forme discontinue. Pour le nom, l'occurrence de l'article est une solution à la neutralisation des oppositions de genre survenue à cause de la disparition du suffixe de genre dans de nombreux cas. Pour l'adjectif, forme qui n'est régulièrement pas précédée de l'article, le genre et le nombre apparaissent dans le cadre de l'accord. Ce phénomène se manifeste non pas par la reprise du suffixe nominal de genre, mais par la surrection de la voyelle [e]. 


\subsection{Le syntagme verbal : la structure du temps et de l'inflexion}

Dans la théorie de la vérification, les traits de la personne et du nombre du sujet vérifient ceux de l'inflexion dont ils occupent le domaine de spécification. (Pollock, 1998 : 113-114) Un point nécessite cependant d'être précisé : la nature du sujet. Les linguistes générativistes, avec lesquels s'accorde Muller, parlent de clitiques sujets. Le statut de ces éléments n'est pas établi de manière définitive. La distinction introduite par Zwicky (1977) entre clitiques simples et clitiques spéciaux a donné lieu à des réflexions visant à appliquer cette typologie aux clitiques du français ainsi qu'à ceux d'autres langues (romanes). La littérature est ainsi parvenue à montrer que les clitiques objets sont spéciaux (les contributions dans Muller 2001b ou Miller et Monachesi 2003). La plupart des études, où l'intérêt pour les clitiques sujets reste relativement faible, démontrent que les clitiques objets sont spéciaux. Au-delà de l'opposition objet vs sujet, Muller (2001a : 77) considère tous les pronoms conjoints comme des clitiques spéciaux. Mais ces éléments, si on leur applique les traits distinctifs établis par Muller, sont loin de présenter les mêmes propriétés morphologiques, phonologiques et syntaxiques. Pour le moment, l'on va se limiter à observer que les clitiques occupent le domaine de spécification de la position tête pour en vérifier les traits. C'est le cas du genre. C'est aussi le cas de l'inflexion, qui est une catégorie de forme discontinue. Les projections du verbe, du temps et de l'inflexion s'organisent de la manière suivante.

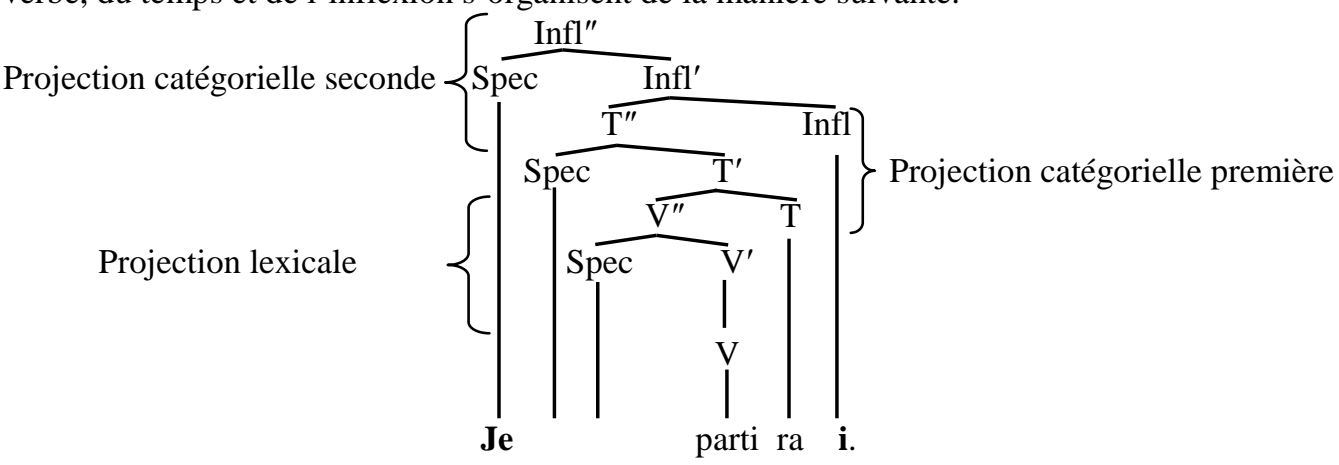

Figure 5 : La structure du syntagme verbal

Comme le nom, le verbe est «bicatégoriel ». Comme le nom, le verbe se caractérise par la vacuité de son domaine de spécification. Ce fait inspire une typologie des classes lexicales. N'est en effet rempli que le domaine de spécification des catégories syntaxiques dépendantes (ou mineures) et « unicatégorielles ». C'est le cas de l'adverbe et de l'adjectif dont le degré constitue la seule catégorie. Le domaine des classes syntaxiques autonomes et «multicatégorielles » est vide, chacune des catégories faisant l'objet d'une projection maximale.

L'observation directe identifie autant de projections maximales que d'items lexicaux. Elle dédie néanmoins quelques projections aux items catégoriels. Suivant cette conception, un item de tout ordre donne lieu à la formalisation d'un syntagme. Certaines raisons qui ont déjà été exposées et d'autres qui le seront amènent à restreindre la dérivation syntagmatique au verbe et au nom. L'inflexion qui représente l'une des deux projections catégorielles du domaine verbal illustre le phénomène de vérification. L'analyse de la dérivation du syntagme nominal vient de révéler que le domaine de spécification de ses projections catégorielles constitutives est occupé par des entités morphologiquement faibles (clitiques et divers items phoriques). L'analyse de la projection maximale I" en donne la confirmation.

À présent qu'est établie la structure du syntagme - domaine catégoriel cohérent élaboré autour d'une classe syntaxique autonome - il faut examiner les modalités de combinaison du domaine verbal et du domaine nominal. La dislocation, que l'on renonce à porter à l'analyse, (Dargnat 2006 : 366) en fournit le cadre. Ce phénomène doit être distingué de ce que la littérature décrit comme le redoublement du sujet lexical. (Auger 1995 et Zribi-Hertz 1994) Le redoublement caractérise le français oral. (Gadet 1991 : 12) D'après les explications de Miller et Monachesi, (2003) la différence de statut entre le redoublement et la dislocation est indiquée à l'écrit par la virgule qui marque une rupture intonative entre la phrase et le syntagme disloqué. Pour le sujet comme pour l'objet, ces indications constitueront difficilement des 
critères objectifs d'analyse. Il est d'ailleurs nécessaire que les faits soient nommés avec précision. Ce qui relève du français oral, c'est la collocation du sujet et du clitique inflexionnel (10) :

L'oncle Joseph, c'est l'aîné, celui qui vend les chaussures, il est court au contraire. (A. Memmi)

Elle, elle s'était métamorphosée pour ses enchantements volontairement, mais moi, qui n'avais été ni enchanté ni victime d'un sort, je demeurais frappé de stupeur devant le fait dont je venais d'être témoin. (Apulée)

Le redoublement, qui est un procédé appositif, opère aussi bien sur le sujet que sur l'objet (11) :

Le producteur revint lui, deux minutes plus tard, l'air satisfait et même réjoui. (F. Sagan)

Tu le connais, l'oncle Joseph ? demandai-je insidieusement. (A. Memmi)

Mais les devis, qui les a tracés ? (Gérard de Nerval)

La dislocation, dont l'application se limite au sujet, résout les problèmes posés par la collocation (12) :

O mon Ennemie, Femme de mon ennemie, où Vache Sauvage est-elle allée ? (R. Kipling)

À quoi cela te sert-il de posséder les étoiles ? (A. de Saint-Exupéry)

Un fait, l'inversion, contribuera à définir avec clarté la dislocation. On distinguera entre l'inversion lexicale et l'inversion clitique. L'inversion clitique concerne précisément le Spéc, Infl', c'està-dire le clitique inflexionnel :

Mais y aura-t-il scrutin de liste ou élection par arrondissement (A. Pitoiset)

Quelle différence y a-t-il donc entre ces deux vases ? (Pasteur Vallerey-Radot)

Elle n'implique en effet le sujet que dans les cas suivants :

- Interrogation totale (14)

Mais peut-être cet animal a-t-il quelque utilité particulière ? (Dino Buzzati)

Le monde, comme la robe du Christ, était-il jeté au sort ? (Châteaubriand)

- Interrogation partielle avec déplacement (à gauche) de l'interrogatif (15) :

Depuis combien de temps ces dômes vivent-ils leur vie secrète ? (Gérard Klein)

Pourquoi donc celui-ci s'altère-t-il quand tandis que celui-là ne s'altère pas ? (Pasteur Vallerey-Radot)

- Interrogation partielle avec effacement (en surface) du clitique inflexionnel (16)

D'où te vient cet accoutrement ? (A. de Musset) $\longrightarrow \quad$ D'où cet accoutrement te vient-il ?

Où est votre mari ? (G. Longelaan) $\longrightarrow$ Où votre mari est-il ?

Il s'agit sans doute de l'application de la règle interdisant la collocation en français standard, c'est-à-dire la juxtaposition du sujet et du clitique inflexionnel. Ainsi, la présence du sujet représente une contrainte d'inversion pour Spéc, Infl'. Si tel est le cas, la différence entre l'assertion et l'interrogation totale réside dans le maintien ou l'effacement en surface (et à droite du verbe) de Spéc, infl'. (17)

Mais (peut-être) cet animal a (-t-il) quelque utilité particulière. (?)

Le monde, comme la robe du Christ, était (-il) jeté au sort. (?)

Cela cesse d'impliquer - comme il est courant dans les approches rectilignes de la syntaxe - le rattachement de l'inflexion au sujet, mais de la projection du nombre du sujet à l'inflexion. La sélection (trait interrompu) assure l'adéquation ou la compatibilité catégorielle (accord ?) du sujet (le nombre et la personne, mais pas le genre) avec l'inflexion du verbe. Cette relation qui s'établit au moyen du clitique inflexionnel, synthèse du nombre du sujet et de la personne du verbe (Cabredo Hofherr 2004 : 103) 
supprime la nécessité de la relation syntagmatique. Il existe une source de blocage de la sélection des traits du sujet : l'emploi non fini (infinitif ou participe) et impersonnel du verbe. Dans ce premier cas, tout élément lexical, indifféremment de sa forme, est capable de jouer le rôle de sujet. Le temps reste une spécificité du verbe, le genre une spécificité du nom. Ce dernier apparaît du reste comme une propriété sans attache syntaxique. Ce n'est pas, en termes de traits, le genre qui élit le sujet à la sélection par l'inflexion, mais uniquement le nombre. En outre, le procès verbal est « extraposé » à l'espace catégoriel sitôt que l'auxiliaire, en cas d'éclatement verbal, le supplée dans le rôle d'hôte du temps et de l'inflexion :

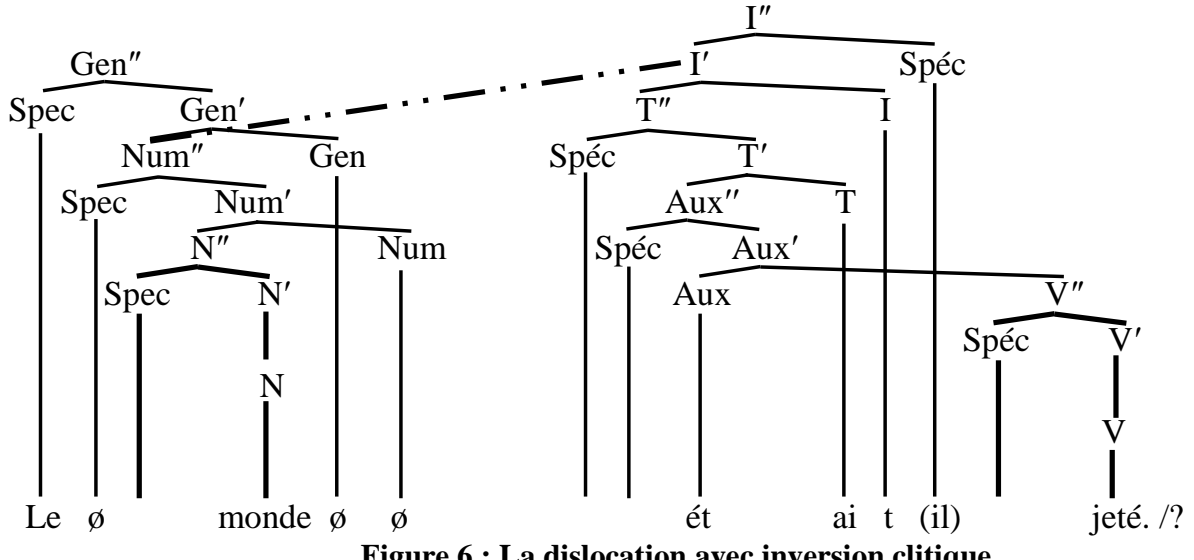

Figure 6 : La dislocation avec inversion clitique

Dans les tours impersonnels du type «Il arrive des gens », il y a en apparence postposition. Cependant, l'absence de sélection du présumé sujet par le clitique inflexionnel est un argument en défaveur de la dislocation. Cette dernière restreint ses applications à des situations précises. Trois faits, de ce point de vue, définissent la dislocation :

- le clitique et la forme pleine sont coréférents ;

- $\quad$ la forme pleine est un argument du verbe, le clitique pas ;

- le clitique et la forme pleine sont nécessairement séparés par l'inflexion.

La dislocation conserve donc la relation de sélection catégorielle de l'inflexion et le statut du clitique inflexionnel est celui d'anaphore ou de cataphore du sujet selon qu'il y a inversion clitique ou postposition du sujet. Ces deux phénomènes constituent en effet une alternative syntaxique. Le maintien du clitique inflexionnel en position préverbale induit en outre la postposition du sujet. C'est pour ce type de dislocation qu'est nécessaire la rupture de l'intonation marquée à l'écrit par la virgule. La relation syntagmatique (trait continu gras) supplée de toute façon la relation structurale de sélection.

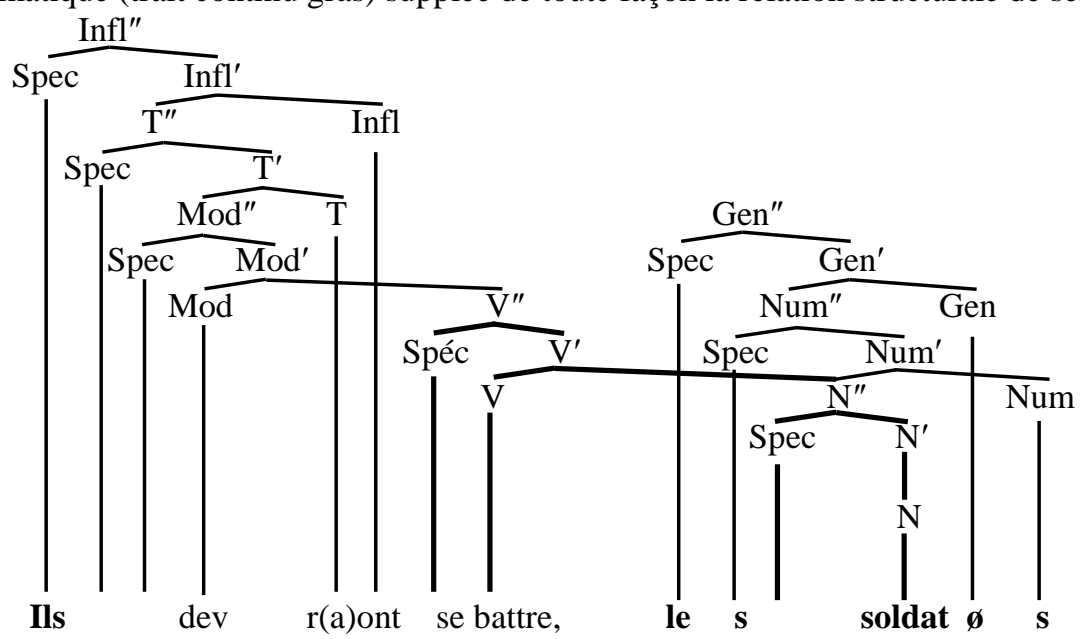

Figure 7 : La dislocation avec postposition du sujet 
Ces indications posent à nouveau la question du statut des clitiques. Tout en reconnaissant leur statut intermédiaire entre mots pleins et affixes, la littérature tend à en faire des affixes. (Miller et Monachesi 2003 ; Muller 2001b : 81; Auger 1995 : 32) Les cas de dislocation où ces éléments sont engagés font même envisager la possibilité de deux sujets. (Muller, 2002) Mais l'impossibilité d'apposer les clitiques aux formes pleines (Grevisse et Goosse 2004), dans les phrases du type « Moi, je chanterai », constitue un argument de poids contre cette analyse. Les clitiques sont surtout exclut du rôle de sujet. (Auger 1995 : 32) À l'évidence, le sujet n'est ni un clitique lexical ni un clitique catégoriel. Il ne peut donc pas s'interpréter comme une propriété catégorielle de l'inflexion. L'absence de position vide fait peser une hypothèque sur l'idée de sa montée syntaxique. Ces raisons expliquent la restriction du rôle de vérification au clitique inflexionnel. Une réponse vient enfin des mouvements syntaxiques qu'Auger exclut de considérer comme un critère décisif de l'analyse. Ainsi, l'affixe (marque temporelle ou désinence verbale) se définit par sa fixité, le clitique par sa mobilité (personne grammaticale) ou par son ellipse (article). Dans ce sens, l'élaboration du syntagme nominal ayant fait état de suffixes du genre, estil abusif, pour caractériser les terminaisons verbales, de parler d'un affixe inflexionnel temporel et d'un affixe inflexionnel ? Mais l'affixe inflexionnel est spécifié, l'affixe temporel ne l'est pas.

\section{Bilan}

Dans ce qui précède, le concept de syntagme bénéficie, contre son émiettement dans la littérature, d'un resserrement avec la description du processus de dérivation du domaine nominal et du domaine verbal. La conception alternative proposée s'avère productive dans l'analyse syntaxique. Elle lui assure l'accès à l'interprétation de structures jugées hors d'atteinte. Elle contribue à décrire le sujet comme l'item lexical d'un système, et sa construction comme la conséquence d'une opération de sélection (catégorielle) et/ou de complémentation (verbale). Le domaine catégoriel du sujet, ainsi que celui du verbe, est souverain. Il ne doit plus guère suffire qu'une tête comporte des positions de compléments pour lui donner le nom de syntagme. Le syntagme, système catégoriel cohérent élaboré autour d'une classe syntaxique autonome (le nom et le verbe), se distingue du groupe en ceci qu'il est régi par l'immanentisme catégoriel. Suivant ce principe, la circulation des catégories du sujet se limite à l'intérieur du syntagme nominal. Celle des catégories verbales est circonscrite à l'intérieur du syntagme verbal. L'inflexion sert, à la fois, de point de jonction et de disjonction entre le nom (sujet) et le verbe. Le rôle de jonction tient à l'amalgame du nombre et de la personne. La disjonction ressortit à l'immanentisme qui consacre respectivement l'exclusivité du genre au domaine du sujet et celle du temps au domaine du verbe. L'intervention dans le domaine de l'un de catégories spécifiques à l'autre constitue une violation syntagmatique (illustrée à l'introduction). La complémentation et la sélection, se substituant à l'équation abstraite $\mathrm{S} \rightarrow \mathrm{SN} \mathrm{SV}$, expliquent les structures au sein desquelles est engagé le sujet. Sur le plan catégoriel, l'inflexion verbale sélectionne le nombre nominal. Sur le plan fonctionnel, le sujet a statut de complément du verbe. Quant à elle, la vérification des traits du verbe incombe au clitique inflexionnel. Finalement, la dislocation offre une approche à la fois intéressante et originale de la construction du sujet.

\section{Références bibliographiques}

Abney S. P. (1987). The English Noun Phrase in its Sentential Aspect. Thèse de doctorat : MIT.

Audet, C-H. (1995). «L'accord du participe passé en une seule règle ». La Structuration conceptuelle du langage. Actes des 9es journées de linguistique. Publication B-201. CIRAL et ICRIP. Faculté des Lettres. Université de Laval : Québec. 165-170.

Auger, J. (1995). «Les clitiques pronominaux en français parlé informel : une approche morphologique. » Revue Québecoise de Linguistique 24 : 13-60.

Baylon, C. et Fabre, P. (1978). Grammaire systématique de la langue française. Paris : Nathan.

Bonnard, H. (1966). Grammaire française. Principes d'une description structurale appliqués à la langue française. Étude normative des formes et de leurs emplois. Dix leçons à la Faculté de Paris-Nanterre (1er cycle). Paris : Jacques Touquet. 
Bonnard, H. (1988). «Verbe et temps ». L'Information grammaticale. 38 : 3-6.

Cabredo Hofherr, P. (2004). «Les clitiques sujets du français et le paramètre du sujet nul ». Langue française 141. Le français parmi les langues romanes. 99-109.

Chomsky, N. (1970). "Remarks on Nominalizations" in Jacobs et Rosenbaum, ed. 184-221 (traduction française : "Remarques sur les nominalisations". In Chomsky, 1975, Questions de sémantique. Paris : Seuil.

Dargnat, M. (2006). L'oral comme fiction. Doctorat de sciences du langage et Ph.D. d'études françaises. Université de Provence et Université de Montréal.

Grevisse, M. et Goosse, A. (2004). Le Bon usage. Bruxelles : De Boeck et Larcier.

Jackendoff, R. (1977). Syntax: a Study of Phrase Structure. Cambridge (Mass): The MIT Press.

Laenzlinger, C. (2006). «Le rôle de l'interface syntaxe-structure informationnelle dans la variation de l'ordre des constituants dans la phrase ». In Nouveaux cahiers de linguistique française 27 : 53-81.

Maingueneau, D. (1994). Syntaxe du français. Paris : Hachette Supérieur.

Miller, P.H. (1992). Clitics and Constituent in Phrase Structure Grammar. New York : Garland (Thèse de doctorat, Université d'Utrecht, 1991).

Muller, C. (2007). «Participe présent, conjonction et construction du sujet ». In Travaux linguistiques du Cerlico, 20, Les formes non finies $d u$ verbe, $2: 19-36$.

Muller, C. (2003). «Evolution de la syntaxe sujet-verbe et réanalyse ». In Verbum XXV-4, 2005, daté 2003. Pp. 481493 (Actes du colloque Diachro-1 de 2002).

Muller, C. (2002). Les bases de la syntaxe. Syntaxe contrastive français-langues voisines. Bordeaux: Presses Universitaires de Bordeaux.

Muller, C. (2001a). « Clitique ou pas ? Une évaluation par degrés ». In Muller, C. (éd.). Clitiques et cliticisation. Paris : Champion.

Muller, C. (2001b). Clitiques et cliticisation. Paris : Champion.

Pollock, J.-Y. (1997). Langage et cognition. Paris : PUF.

Riegel, M. et al. (1994). Grammaire méthodique du français. Paris : PUF.

Zribi-Hertz, A. (1994). "The syntax of nominative clitics in Standard and Advanced French.” In Guiglielmo Cinque, Jan Koster, Jean-Yves Pollock, Luigi Rizzi et Rafaella Zanuttini, éd. Paths towards Universal Grammar: Studies in Honor of Richard S. Kayne. Washington D.C.: Georgetown University Press. 453-472.

Zwicky, A. M. et Pullum, G. K. (1983). “Cliticization vs. Inflection: English n't”. Language 59.3 : 502-513.

Zwicky, A. (1977). On clitics. Bloomington: Indiana Linguistics Club.

\footnotetext{
${ }^{1}$ Parler de formes verbales composées, comme le font les grammaires, suppose une différence de constituants. Une telle différence existe-t-elle ? L'item verbal est plutôt de forme éclatée, associant l'auxiliaire et le verbe auxilié.

${ }^{2}$ Résultatif ou statif, le passif n'est pas un verbe, mais un véritable adjectif. Au moins deux faits expliquent l'accord : la fonction attribut, le statut copulatif de être.

${ }^{3}$ «Les catégories grammaticales sont toujours relatives à une langue donnée à une certaine période de l'histoire de chaque langue. »

${ }^{4}$ Muller (2002), qui estime que l'article manque d'équivalent synchronique, l'exclut des clitiques. La cliticisation procède en effet d'un affaiblissement morphologique dû soit à un effacement, soit à une réduction. L'idée qu'on s'en fait ici est que l'article représente le clitique de ces suffixes de genre (latins ?) dont on ne trouve aujourd'hui que des survivances et en nombre fort limité.

${ }^{5}$ Bonnard pense que la récurrence des marques l'opposition masculin/féminin, c'est-à-dire la catégorie du genre cimente le groupe nominal. (1966:7)
} 